Acta Crystallographica Section E

Structure Reports

Online

ISSN 1600-5368

\section{4-(1H-Pyrrolo[2,3-b]pyridin-2-yl)- pyridine}

\section{Ping-Hsin Huang, ${ }^{a *}$ Yuh-Sheng Wen ${ }^{b}$ and Jiun-Yi Shen ${ }^{c}$}

${ }^{a}$ Cardinal Tien College of Healthcare \& Management, Taipei, Taiwan 231, Republic of China, ${ }^{\mathbf{b}}$ Institute of Chemistry, Academia Sinica, Nankang, Taipei, Taiwan, Republic of China, and ' Department of Chemistry, National Taiwan University, Taipei, Taiwan, Republic of China

Correspondence e-mail: pshuang@ctcn.edu.tw

Received 12 March 2013; accepted 29 March 2013

Key indicators: single-crystal X-ray study; $T=295 \mathrm{~K}$; mean $\sigma(\mathrm{C}-\mathrm{C})=0.003 \AA$; $R$ factor $=0.058 ; w R$ factor $=0.124 ;$ data-to-parameter ratio $=12.3$.

The asymmetric unit of the title compound, $\mathrm{C}_{12} \mathrm{H}_{9} \mathrm{~N}_{3}$, contains two independent molecules in which the dihedral angle between the pyridine and azaindole rings are 8.23 (6) and $9.89(2)^{\circ}$. In the crystal, both types of molecule are connected by pairs of $\mathrm{N}-\mathrm{H}-\mathrm{N}$ hydrogen bonds into inversion dimers.

\section{Related literature}

For the production of luminescent organic compounds, see: Liu et al. (2000); Parcerisa et al. (2008). For related structures, see: Huang et al. (2012).

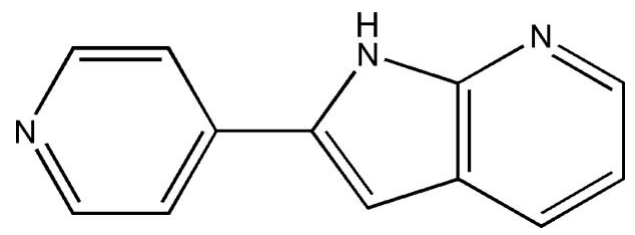

\section{Experimental}

Crystal data

$\mathrm{C}_{12} \mathrm{H}_{9} \mathrm{~N}_{3}$

$M_{r}=195.22$

Triclinic, $P \overline{1}$

$$
\begin{aligned}
& \alpha=83.372(2)^{\circ} \\
& \beta=86.697(2)^{\circ} \\
& \gamma=87.427(2)^{\circ} \\
& V=947.69(13) \AA^{3} \\
& Z=4
\end{aligned}
$$

Data collection

Bruker SMART APEX CCD areadetector diffractometer

Absorption correction: multi-scan (SADABS; Bruker, 2001)

$T_{\min }=0.975, T_{\max }=0.996$

\section{Refinement}

$R\left[F^{2}>2 \sigma\left(F^{2}\right)\right]=0.058$

$w R\left(F^{2}\right)=0.124$

$S=1.14$

3329 reflections
Mo $K \alpha$ radiation

$\mu=0.09 \mathrm{~mm}^{-1}$

$T=295 \mathrm{~K}$

$0.30 \times 0.20 \times 0.05 \mathrm{~mm}$

10193 measured reflections 3329 independent reflections 2573 reflections with $I>2 \sigma(I)$ $R_{\text {int }}=0.034$

Table 1

Hydrogen-bond geometry $\left(\AA{ }^{\circ}\right)$.

\begin{tabular}{lllll}
\hline$D-\mathrm{H} \cdots A$ & $D-\mathrm{H}$ & $\mathrm{H} \cdots A$ & $D \cdots A$ & $D-\mathrm{H} \cdots A$ \\
\hline $\mathrm{N} 1-\mathrm{H} 1 \cdots \mathrm{N} 2^{\mathrm{i}}$ & 0.86 & 2.22 & $3.061(3)$ & 167 \\
$\mathrm{~N} 4-\mathrm{H} 4 A \cdots \mathrm{N} 5^{\mathrm{ii}}$ & 0.86 & 2.22 & $3.066(3)$ & 169 \\
\hline
\end{tabular}

Symmetry codes: (i) $-x,-y+2,-z+2$; (ii) $-x+1,-y+2,-z+1$.

Data collection: SMART (Bruker, 2001); cell refinement: SAINT (Bruker, 2001); data reduction: SAINT; program(s) used to solve structure: SHELXS97 (Sheldrick, 2008); program(s) used to refine structure: SHELXL97 (Sheldrick, 2008); molecular graphics: ORTEP-3 for Windows (Farrugia, 2012); software used to prepare material for publication: Win $G X$ (Farrugia, 2012).

This work is partially supported by the instrumentation center, National Taiwan University, and Cardinal Tien College of Healthcare \& Management.

Supplementary data and figures for this paper are available from the IUCr electronic archives (Reference: NC2308).

\title{
References
}

Bruker (2001). SMART, SAINT and SADABS. Bruker AXS Inc., Madison, Wisconsin, USA.

Farrugia, L. J. (2012). J. Appl. Cryst. 45, 849-854.

Huang, P.-H., Wen, Y.-S. \& Shen, J.-Y. (2012). Acta Cryst. E68, o1943.

Liu, S. F., Wu, Q., Schmider, H. L., Aziz, H., Hu, N. X., Popovic, Z. \& Wang, S. (2000). J. Am. Chem. Soc. 122, 3671-3678.

Parcerisa, J., Romero, M. \& Pujol, M. D. (2008). Tetrahedron, 64, 500-507.

Sheldrick, G. M. (2008). Acta Cryst. A64, 112-122. 


\section{supporting information}

Acta Cryst. (2013). E69, o674 [https://doi.org/10.1107/S1600536813008672]

\section{4-(1H-Pyrrolo[2,3-b] pyridin-2-yl)pyridine}

\section{Ping-Hsin Huang, Yuh-Sheng Wen and Jiun-Yi Shen}

\section{S1. Comment}

The title compound has been shown to be an precursor for the production of luminescent organic compound (Liu et al., 2000). In the crystal structure of the title compound two crystallographically independent molecules are found which shows no large structural differences. Both molecules are nearly coplanar, the dihedral angles between the pyridine and the azaindole rings is $8.23(6)^{\circ}$ and $9.89(2)^{\circ}$ (Huang et al., 2012). Each of these molecules is connected into centrosymmetrically dimers by intermolecular $\mathrm{N}-\mathrm{H}-\mathrm{N}$ hydrogen bonding.

\section{S2. Experimental}

The compound was synthesized by the following procedure (Parcerisa et al., 2008). A solution of [3-(2-hydroxy-2pyridin-4-yl-ethyl)-pyridin-2-yl]-carbamic acid tert-butyl ester $(1 \mathrm{mmol}$ and acetonitrile $(12 \mathrm{ml})$ was cooled to ice temperature. Afterwards triethylamine $(1.2 \mathrm{mmol})$ and trifluoromethanesulfonic anhydride $(1.1 \mathrm{mmol})$ was added over a period of $5 \mathrm{~min}$. The mixture was stirred at room temperature for $2 \mathrm{~h}$, trifluoroacetic acid was added (1.5 mmol) and afterwards the mixture was heated under reflux for $1 \mathrm{~h}$. The mixture was cooled to room temperature and neutralized using $2 \mathrm{~N} \mathrm{NaOH}$. The aqueous layer was extracted with ethyl ether and the organic extract was washed with brine and aqueous $\mathrm{Na}_{2} \mathrm{SO}_{4}$, dried and concentrated. The residue was purified by column chromatography using hexane/ethyl acetate (2:8) as eluent, followed by recrystallization in $\mathrm{CH}_{2} \mathrm{Cl}_{2}$ and hexane to give a white solid in 64\% yield. Crystals suitable for X-ray diffraction were grown from a $\mathrm{CH}_{2} \mathrm{Cl}_{2}$ solution layered with hexane at room temperature. ${ }^{1} \mathrm{H} \mathrm{NMR}\left(\mathrm{CDCl}_{3}, 300\right.$ MHz): 8.62 (dd, 2 H, J= 1.0, 3.1 Hz), 8.29 (dd, 1 H, J=1.0, 3.4 Hz), 8.00 (dd, 1 H, J = 1.0, $5.3 \mathrm{~Hz}), 7.72$ (dd, $2 \mathrm{H}, \mathrm{J}=1.0$, $3.1 \mathrm{~Hz}), 7.13(\mathrm{dd}, 1 \mathrm{H}, \mathrm{J}=3.4,5.3 \mathrm{~Hz}), 6.99(\mathrm{~s}, 1 \mathrm{H})$, Anal. Calcd for $\mathrm{C}_{12} \mathrm{H}_{9} \mathrm{~N}_{3}: \mathrm{C}, 73.83 ; \mathrm{H}, 4.65 ; \mathrm{N}, 21.52$. Found: $\mathrm{C}$, 74.21; H, 4.40; N, 21.34 .

\section{S3. Refinement}

$\mathrm{H}$ atoms were located in difference map but were positioned with idealized geometry and refined isotropic with $U_{\text {iso }}(\mathrm{H})=$ $1.2 U_{\mathrm{eq}}(\mathrm{C}, \mathrm{N})$. 


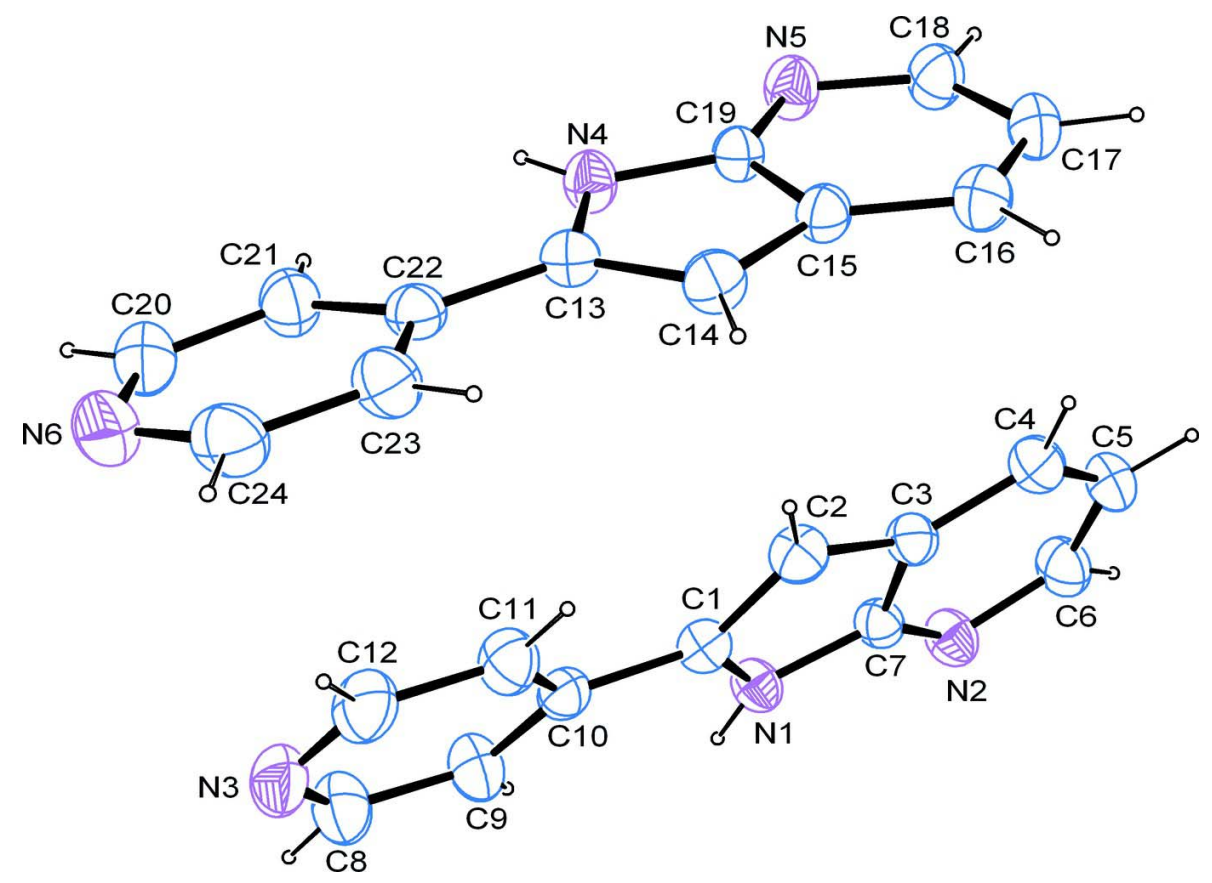

Figure 1

Molecular structure of the title compound with labeling and displacement ellipsoids drawn at the $30 \%$ probability level.

$\mathrm{H}$ atoms are shown as small spheres of arbitrary radii.

4-(1H-Pyrrolo[2,3-b]pyridin-2-yl)pyridine

Crystal data

$\mathrm{C}_{12} \mathrm{H}_{9} \mathrm{~N}_{3}$

$M_{r}=195.22$

Triclinic, $P \overline{1}$

Hall symbol: -P 1

$a=6.5529(5) \AA$

$b=10.0457(8) \AA$

$c=14.5282(11) \AA$

$\alpha=83.372(2)^{\circ}$

$\beta=86.697(2)^{\circ}$

$\gamma=87.427(2)^{\circ}$

$V=947.69(13) \AA^{3}$

$Z=4$

\section{Data collection}

Bruker SMART APEX CCD area-detector diffractometer

Radiation source: fine-focus sealed tube

Graphite monochromator

$\omega$ scans

Absorption correction: multi-scan

(SADABS; Bruker, 2001)

$T_{\min }=0.975, T_{\max }=0.996$
$F(000)=408$

$D_{\mathrm{x}}=1.368 \mathrm{Mg} \mathrm{m}^{-3}$

$D_{\mathrm{m}}=1.368 \mathrm{Mg} \mathrm{m}^{-3}$

$D_{\mathrm{m}}$ measured by not measured

Mo $K \alpha$ radiation, $\lambda=0.71073 \AA$

Cell parameters from 1585 reflections

$\theta=2.6-23.3^{\circ}$

$\mu=0.09 \mathrm{~mm}^{-1}$

$T=295 \mathrm{~K}$

Plate, colorless

$0.30 \times 0.20 \times 0.05 \mathrm{~mm}$

10193 measured reflections

3329 independent reflections

2573 reflections with $I>2 \sigma(I)$

$R_{\text {int }}=0.034$

$\theta_{\text {max }}=25.0^{\circ}, \theta_{\text {min }}=1.4^{\circ}$

$h=-7 \rightarrow 7$

$k=-11 \rightarrow 11$

$l=-17 \rightarrow 17$ 


\section{Refinement}

Refinement on $F^{2}$

Least-squares matrix: full

$R\left[F^{2}>2 \sigma\left(F^{2}\right)\right]=0.058$

$w R\left(F^{2}\right)=0.124$

$S=1.14$

3329 reflections

271 parameters

0 restraints

Primary atom site location: structure-invariant direct methods
Secondary atom site location: difference Fourier map

Hydrogen site location: inferred from neighbouring sites

$\mathrm{H}$-atom parameters constrained

$w=1 /\left[\sigma^{2}\left(F_{\mathrm{o}}^{2}\right)+(0.0442 P)^{2}+0.126 P\right]$ where $P=\left(F_{\mathrm{o}}{ }^{2}+2 F_{\mathrm{c}}{ }^{2}\right) / 3$

$(\Delta / \sigma)_{\max }<0.001$

$\Delta \rho_{\max }=0.14 \mathrm{e} \AA^{-3}$

$\Delta \rho_{\min }=-0.17$ e $\AA^{-3}$

Special details

Geometry. All e.s.d.'s (except the e.s.d. in the dihedral angle between two l.s. planes) are estimated using the full covariance matrix. The cell e.s.d.'s are taken into account individually in the estimation of e.s.d.'s in distances, angles and torsion angles; correlations between e.s.d.'s in cell parameters are only used when they are defined by crystal symmetry. An approximate (isotropic) treatment of cell e.s.d.'s is used for estimating e.s.d.'s involving 1.s. planes.

Refinement. Refinement of $F^{2}$ against ALL reflections. The weighted $R$-factor $w R$ and goodness of fit $S$ are based on $F^{2}$, conventional $R$-factors $R$ are based on $F$, with $F$ set to zero for negative $F^{2}$. The threshold expression of $F^{2}>\sigma\left(F^{2}\right)$ is used only for calculating $R$-factors(gt) etc. and is not relevant to the choice of reflections for refinement. $R$-factors based on $F^{2}$ are statistically about twice as large as those based on $F$, and $R$ - factors based on ALL data will be even larger.

Fractional atomic coordinates and isotropic or equivalent isotropic displacement parameters $\left(\AA^{2}\right)$

\begin{tabular}{|c|c|c|c|c|}
\hline & $x$ & $y$ & $z$ & $U_{\text {iso }} * / U_{\text {eq }}$ \\
\hline N1 & $0.2449(3)$ & $0.99700(17)$ & $0.92427(12)$ & $0.0443(5)$ \\
\hline H1 & 0.1787 & 1.0568 & 0.9532 & $0.053 *$ \\
\hline N2 & -0.0037 & $0.82637(19)$ & $0.94609(13)$ & $0.0523(5)$ \\
\hline N3 & $0.7664(4)$ & $1.3698(2)$ & $0.86462(16)$ & $0.0704(6)$ \\
\hline N4 & $0.7252(3)$ & $0.97632(17)$ & $0.57580(12)$ & $0.0452(5)$ \\
\hline $\mathrm{H} 4 \mathrm{~A}$ & 0.6651 & 1.0437 & 0.5450 & $0.054 *$ \\
\hline N5 & $0.4869(3)$ & 0.80868 (19) & $0.55706(13)$ & $0.0524(5)$ \\
\hline N6 & $1.2240(4)$ & $1.3404(2)$ & $0.62691(16)$ & $0.0710(6)$ \\
\hline $\mathrm{C} 1$ & $0.4379(3)$ & $1.0098(2)$ & $0.88094(14)$ & $0.0423(5)$ \\
\hline $\mathrm{C} 2$ & $0.4911(3)$ & $0.8951(2)$ & $0.84219(15)$ & $0.0493(6)$ \\
\hline $\mathrm{H} 2$ & 0.6131 & 0.8780 & 0.8089 & $0.059 *$ \\
\hline $\mathrm{C} 3$ & 0.3285 & $0.8067(2)$ & $0.86152(15)$ & $0.0435(5)$ \\
\hline $\mathrm{C} 4$ & $0.2881(4)$ & $0.6784(2)$ & $0.84216(16)$ & $0.0548(6)$ \\
\hline $\mathrm{H} 4$ & 0.3826 & 0.6290 & 0.8083 & $0.066^{*}$ \\
\hline $\mathrm{C} 5$ & 0.1035 (4) & $0.6273(2)$ & $0.87473(17)$ & $0.0576(7)$ \\
\hline H5 & 0.0713 & 0.5418 & 0.8630 & $0.069^{*}$ \\
\hline C6 & $-0.0344(4)$ & $0.7025(2)$ & $0.92484(17)$ & $0.0589(7)$ \\
\hline H6 & -0.1580 & 0.6644 & 0.9454 & $0.071 *$ \\
\hline $\mathrm{C} 7$ & $0.1773(3)$ & $0.8734(2)$ & $0.91330(14)$ & $0.0416(5)$ \\
\hline $\mathrm{C} 8$ & $0.5755(5)$ & $1.3615(3)$ & 0.90108 (19) & $0.0712(8)$ \\
\hline H8 & 0.5140 & 1.4376 & 0.9232 & $0.085^{*}$ \\
\hline C9 & $0.4636(4)$ & $1.2481(2)$ & $0.90816(18)$ & $0.0603(7)$ \\
\hline $\mathrm{H} 9$ & 0.3301 & 1.2493 & 0.9337 & $0.072 *$ \\
\hline $\mathrm{C} 10$ & $0.5497(3)$ & $1.1326(2)$ & $0.87731(14)$ & $0.0445(5)$ \\
\hline $\mathrm{C} 11$ & $0.7495(4)$ & $1.1396(2)$ & $0.84044(16)$ & $0.0561(6)$ \\
\hline
\end{tabular}




$\begin{array}{lllll}\mathrm{H} 11 & 0.8162 & 1.0644 & 0.8193 & 0.067^{*} \\ \mathrm{C} 12 & 0.8479(4) & 1.2578(3) & 0.83527(18) & 0.0678(7) \\ \mathrm{H} 12 & 0.9811 & 1.2597 & 0.8094 & 0.081^{*} \\ \mathrm{C} 13 & 0.9063(3) & 0.9796(2) & 0.61960(14) & 0.0424(5) \\ \mathrm{C} 14 & 0.9524(3) & 0.8542(2) & 0.66219(15) & 0.0485(6) \\ \mathrm{H} 14 & 1.0656 & 0.8293 & 0.6969 & 0.058^{*} \\ \mathrm{C} 15 & 0.7970(3) & 0.7688(2) & 0.64396(14) & 0.0436(5) \\ \mathrm{C} 16 & 0.7536(4) & 0.6348(2) & 0.66723(16) & 0.0554(6) \\ \mathrm{H} 16 & 0.8395 & 0.5771 & 0.7035 & 0.066^{*} \\ \mathrm{C} 17 & 0.5792(4) & 0.5904(2) & 0.63473(17) & 0.0573(6) \\ \mathrm{H} 17 & 0.5454 & 0.5012 & 0.6486 & 0.069^{*} \\ \mathrm{C} 18 & 0.4535(4) & 0.6788(2) & 0.58133(17) & 0.0557(6) \\ \mathrm{H} 18 & 0.3366 & 0.6450 & 0.5607 & 0.067^{*} \\ \mathrm{C} 19 & 0.6574(3) & 0.8490(2) & 0.58945(14) & 0.0415(5) \\ \mathrm{C} 20 & 1.0412(5) & 1.3402(3) & 0.59264(18) & 0.0692(8) \\ \mathrm{H} 20 & 0.9818 & 1.4225 & 0.5701 & 0.083^{*} \\ \mathrm{C} 21 & 0.9327(4) & 1.2275(2) & 0.58795(16) & 0.0566(6) \\ \mathrm{H} 21 & 0.8044 & 1.2350 & 0.5631 & 0.068^{*} \\ \mathrm{C} 22 & 1.0152(3) & 1.1027(2) & 0.62031(14) & 0.0444(5) \\ \mathrm{C} 23 & 1.2075(4) & 1.1013(2) & 0.65608(17) & 0.0559(6) \\ \mathrm{H} 23 & 1.2712 & 1.0205 & 0.6787 & 0.067^{*} \\ \text { C24 } & 1.3034(4) & 1.2199(3) & 0.65792(18) & 0.0672(7) \\ \mathrm{H} 24 & 1.4319 & 1.2159 & 0.6824 & 0.081^{*} \\ & & & & \end{array}$

Atomic displacement parameters $\left(\AA^{2}\right)$

\begin{tabular}{lllllll}
\hline & $U^{11}$ & $U^{22}$ & $U^{33}$ & $U^{12}$ & $U^{13}$ & $U^{23}$ \\
\hline $\mathrm{N} 1$ & $0.0419(10)$ & $0.0413(11)$ & $0.0508(11)$ & $-0.0013(8)$ & $0.0011(9)$ & $-0.0113(8)$ \\
$\mathrm{N} 2$ & $0.0454(11)$ & $0.0507(12)$ & $0.0623(13)$ & $-0.0102(9)$ & $0.0003(9)$ & $-0.0112(9)$ \\
$\mathrm{N} 3$ & $0.0765(16)$ & $0.0626(16)$ & $0.0714(15)$ & $-0.0247(13)$ & $-0.0063(12)$ & $0.0053(12)$ \\
$\mathrm{N} 4$ & $0.0435(11)$ & $0.0392(11)$ & $0.0524(11)$ & $-0.0022(8)$ & $-0.0088(9)$ & $-0.0003(8)$ \\
$\mathrm{N} 5$ & $0.0511(12)$ & $0.0480(12)$ & $0.0583(12)$ & $-0.0112(9)$ & $-0.0108(9)$ & $0.0003(9)$ \\
$\mathrm{N} 6$ & $0.0781(17)$ & $0.0669(16)$ & $0.0707(15)$ & $-0.0277(13)$ & $0.0007(13)$ & $-0.0125(12)$ \\
$\mathrm{C} 1$ & $0.0354(12)$ & $0.0475(14)$ & $0.0435(13)$ & $-0.0009(10)$ & $-0.0020(10)$ & $-0.0034(10)$ \\
$\mathrm{C} 2$ & $0.0432(13)$ & $0.0539(15)$ & $0.0498(14)$ & $0.0005(11)$ & $0.0038(11)$ & $-0.0054(11)$ \\
$\mathrm{C} 3$ & $0.0457(13)$ & $0.0409(13)$ & $0.0441(13)$ & $0.0020(10)$ & $-0.0037(10)$ & $-0.0057(10)$ \\
$\mathrm{C} 4$ & $0.0627(16)$ & $0.0474(15)$ & $0.0542(15)$ & $0.0029(12)$ & $0.0004(12)$ & $-0.0089(11)$ \\
$\mathrm{C} 5$ & $0.0703(17)$ & $0.0414(14)$ & $0.0629(16)$ & $-0.0094(12)$ & $-0.0066(13)$ & $-0.0096(11)$ \\
$\mathrm{C} 6$ & $0.0570(15)$ & $0.0540(16)$ & $0.0674(17)$ & $-0.0163(12)$ & $-0.0014(13)$ & $-0.0095(13)$ \\
$\mathrm{C} 7$ & $0.0408(12)$ & $0.0412(13)$ & $0.0439(13)$ & $-0.0038(10)$ & $-0.0064(10)$ & $-0.0062(10)$ \\
C8 & $0.080(2)$ & $0.0510(17)$ & $0.083(2)$ & $-0.0045(14)$ & $-0.0019(16)$ & $-0.0076(14)$ \\
C9 & $0.0568(15)$ & $0.0499(16)$ & $0.0747(18)$ & $-0.0087(12)$ & $0.0038(13)$ & $-0.0109(13)$ \\
C10 & $0.0477(13)$ & $0.0461(14)$ & $0.0394(12)$ & $-0.0052(10)$ & $-0.0064(10)$ & $0.0002(10)$ \\
C11 & $0.0500(14)$ & $0.0573(16)$ & $0.0607(16)$ & $-0.0104(12)$ & $0.0045(12)$ & $-0.0049(12)$ \\
C12 & $0.0593(17)$ & $0.076(2)$ & $0.0665(18)$ & $-0.0216(15)$ & $0.0066(13)$ & $0.0018(15)$ \\
C13 & $0.0394(12)$ & $0.0457(14)$ & $0.0423(12)$ & $-0.0011(10)$ & $-0.0029(10)$ & $-0.0059(10)$ \\
C14 & $0.0448(13)$ & $0.0499(15)$ & $0.0509(14)$ & $0.0019(11)$ & $-0.0111(11)$ & $-0.0034(11)$ \\
C15 & $0.0465(13)$ & $0.0395(13)$ & $0.0439(13)$ & $0.0006(10)$ & $-0.0027(10)$ & $-0.0016(10)$
\end{tabular}




\begin{tabular}{lllllll}
\hline C16 & $0.0632(16)$ & $0.0461(15)$ & $0.0559(15)$ & $0.0016(12)$ & $-0.0070(12)$ & $-0.0009(11)$ \\
C17 & $0.0707(17)$ & $0.0407(14)$ & $0.0604(16)$ & $-0.0123(12)$ & $-0.0045(13)$ & $-0.0010(11)$ \\
C18 & $0.0556(15)$ & $0.0524(16)$ & $0.0595(15)$ & $-0.0157(12)$ & $-0.0078(12)$ & $-0.0004(12)$ \\
C19 & $0.0431(12)$ & $0.0385(13)$ & $0.0429(12)$ & $-0.0062(10)$ & $-0.0006(10)$ & $-0.0040(9)$ \\
C20 & $0.087(2)$ & $0.0538(17)$ & $0.0668(18)$ & $-0.0156(15)$ & $-0.0081(16)$ & $0.0007(13)$ \\
C21 & $0.0595(15)$ & $0.0505(15)$ & $0.0601(16)$ & $-0.0079(12)$ & $-0.0115(12)$ & $-0.0013(12)$ \\
C22 & $0.0440(13)$ & $0.0497(14)$ & $0.0403(13)$ & $-0.0053(10)$ & $0.0007(10)$ & $-0.0083(10)$ \\
C23 & $0.0494(14)$ & $0.0582(16)$ & $0.0618(16)$ & $-0.0072(12)$ & $-0.0079(12)$ & $-0.0093(12)$ \\
C24 & $0.0570(16)$ & $0.082(2)$ & $0.0658(18)$ & $-0.0206(15)$ & $-0.0063(13)$ & $-0.0156(15)$ \\
\hline
\end{tabular}

Geometric parameters $\left(\AA,{ }^{\circ}\right)$

\begin{tabular}{|c|c|c|c|}
\hline $\mathrm{N} 1-\mathrm{C} 7$ & $1.366(2)$ & $\mathrm{C} 8-\mathrm{H} 8$ & 0.9300 \\
\hline $\mathrm{N} 1-\mathrm{C} 1$ & $1.384(2)$ & $\mathrm{C} 9-\mathrm{C} 10$ & $1.378(3)$ \\
\hline $\mathrm{N} 1-\mathrm{H} 1$ & 0.8600 & $\mathrm{C} 9-\mathrm{H} 9$ & 0.9300 \\
\hline $\mathrm{N} 2-\mathrm{C} 7$ & $1.338(3)$ & $\mathrm{C} 10-\mathrm{C} 11$ & $1.387(3)$ \\
\hline $\mathrm{N} 2-\mathrm{C} 6$ & $1.343(3)$ & $\mathrm{C} 11-\mathrm{C} 12$ & $1.368(3)$ \\
\hline $\mathrm{N} 3-\mathrm{C} 12$ & $1.329(3)$ & $\mathrm{C} 11-\mathrm{H} 11$ & 0.9300 \\
\hline $\mathrm{N} 3-\mathrm{C} 8$ & $1.332(3)$ & $\mathrm{C} 12-\mathrm{H} 12$ & 0.9300 \\
\hline $\mathrm{N} 4-\mathrm{C} 19$ & $1.362(3)$ & $\mathrm{C} 13-\mathrm{C} 14$ & $1.367(3)$ \\
\hline $\mathrm{N} 4-\mathrm{C} 13$ & $1.382(2)$ & $\mathrm{C} 13-\mathrm{C} 22$ & $1.457(3)$ \\
\hline $\mathrm{N} 4-\mathrm{H} 4 \mathrm{~A}$ & 0.8600 & $\mathrm{C} 14-\mathrm{C} 15$ & $1.416(3)$ \\
\hline $\mathrm{N} 5-\mathrm{C} 19$ & $1.334(3)$ & $\mathrm{C} 14-\mathrm{H} 14$ & 0.9300 \\
\hline $\mathrm{N} 5-\mathrm{C} 18$ & $1.336(3)$ & $\mathrm{C} 15-\mathrm{C} 16$ & $1.388(3)$ \\
\hline N6-C20 & 1.324 & $\mathrm{C} 15-\mathrm{C} 19$ & $1.408(3)$ \\
\hline $\mathrm{N} 6-\mathrm{C} 24$ & $1.336(3)$ & $\mathrm{C} 16-\mathrm{C} 17$ & $1.373(3)$ \\
\hline $\mathrm{C} 1-\mathrm{C} 2$ & $1.362(3)$ & $\mathrm{C} 16-\mathrm{H} 16$ & 0.9300 \\
\hline $\mathrm{C} 1-\mathrm{C} 10$ & $1.457(3)$ & $\mathrm{C} 17-\mathrm{C} 18$ & $1.384(3)$ \\
\hline $\mathrm{C} 2-\mathrm{C} 3$ & $1.413(3)$ & $\mathrm{C} 17-\mathrm{H} 17$ & 0.9300 \\
\hline $\mathrm{C} 2-\mathrm{H} 2$ & 0.9300 & $\mathrm{C} 18-\mathrm{H} 18$ & 0.9300 \\
\hline $\mathrm{C} 3-\mathrm{C} 4$ & $1.390(3)$ & $\mathrm{C} 20-\mathrm{C} 21$ & $1.374(3)$ \\
\hline $\mathrm{C} 3-\mathrm{C} 7$ & $1.403(3)$ & $\mathrm{C} 20-\mathrm{H} 20$ & 0.9300 \\
\hline $\mathrm{C} 4-\mathrm{C} 5$ & $1.373(3)$ & $\mathrm{C} 21-\mathrm{C} 22$ & $1.384(3)$ \\
\hline $\mathrm{C} 4-\mathrm{H} 4$ & 0.9300 & $\mathrm{C} 21-\mathrm{H} 21$ & 0.9300 \\
\hline $\mathrm{C} 5-\mathrm{C} 6$ & $1.381(3)$ & $\mathrm{C} 22-\mathrm{C} 23$ & $1.390(3)$ \\
\hline $\mathrm{C} 5-\mathrm{H} 5$ & 0.9300 & $\mathrm{C} 23-\mathrm{C} 24$ & $1.375(3)$ \\
\hline C6- 66 & 0.9300 & $\mathrm{C} 23-\mathrm{H} 23$ & 0.9300 \\
\hline $\mathrm{C} 8-\mathrm{C} 9$ & $1.373(3)$ & $\mathrm{C} 24-\mathrm{H} 24$ & 0.9300 \\
\hline $\mathrm{C} 7-\mathrm{N} 1-\mathrm{C} 1$ & $108.49(17)$ & $\mathrm{C} 12-\mathrm{C} 11-\mathrm{H} 11$ & 120.1 \\
\hline $\mathrm{C} 7-\mathrm{N} 1-\mathrm{H} 1$ & 125.8 & $\mathrm{C} 10-\mathrm{C} 11-\mathrm{H} 11$ & 120.1 \\
\hline $\mathrm{C} 1-\mathrm{N} 1-\mathrm{H} 1$ & 125.8 & $\mathrm{~N} 3-\mathrm{C} 12-\mathrm{C} 11$ & $124.5(3)$ \\
\hline $\mathrm{C} 7-\mathrm{N} 2-\mathrm{C} 6$ & $113.4(2)$ & $\mathrm{N} 3-\mathrm{C} 12-\mathrm{H} 12$ & 117.7 \\
\hline $\mathrm{C} 12-\mathrm{N} 3-\mathrm{C} 8$ & $115.3(2)$ & $\mathrm{C} 11-\mathrm{C} 12-\mathrm{H} 12$ & 117.7 \\
\hline $\mathrm{C} 19-\mathrm{N} 4-\mathrm{C} 13$ & $108.82(17)$ & $\mathrm{C} 14-\mathrm{C} 13-\mathrm{N} 4$ & $108.76(19)$ \\
\hline $\mathrm{C} 19-\mathrm{N} 4-\mathrm{H} 4 \mathrm{~A}$ & 125.6 & $\mathrm{C} 14-\mathrm{C} 13-\mathrm{C} 22$ & $128.8(2)$ \\
\hline $\mathrm{C} 13-\mathrm{N} 4-\mathrm{H} 4 \mathrm{~A}$ & 125.6 & $\mathrm{~N} 4-\mathrm{C} 13-\mathrm{C} 22$ & $122.39(19)$ \\
\hline $\mathrm{C} 19-\mathrm{N} 5-\mathrm{C} 18$ & $113.62(19)$ & $\mathrm{C} 13-\mathrm{C} 14-\mathrm{C} 15$ & 107.79 (19) \\
\hline
\end{tabular}




\begin{tabular}{|c|c|c|c|}
\hline $\mathrm{C} 20-\mathrm{N} 6-\mathrm{C} 24$ & $115.3(2)$ & $\mathrm{C} 13-\mathrm{C} 14-\mathrm{H} 14$ & 126.1 \\
\hline $\mathrm{C} 2-\mathrm{C} 1-\mathrm{N} 1$ & $108.75(19)$ & $\mathrm{C} 15-\mathrm{C} 14-\mathrm{H} 14$ & 126.1 \\
\hline $\mathrm{C} 2-\mathrm{C} 1-\mathrm{C} 10$ & $129.1(2)$ & $\mathrm{C} 16-\mathrm{C} 15-\mathrm{C} 19$ & $117.3(2)$ \\
\hline $\mathrm{N} 1-\mathrm{C} 1-\mathrm{C} 10$ & $122.06(19)$ & $\mathrm{C} 16-\mathrm{C} 15-\mathrm{C} 14$ & $136.3(2)$ \\
\hline $\mathrm{C} 1-\mathrm{C} 2-\mathrm{C} 3$ & $108.01(19)$ & $\mathrm{C} 19-\mathrm{C} 15-\mathrm{C} 14$ & $106.40(18)$ \\
\hline $\mathrm{C} 1-\mathrm{C} 2-\mathrm{H} 2$ & 126.0 & $\mathrm{C} 17-\mathrm{C} 16-\mathrm{C} 15$ & $117.6(2)$ \\
\hline $\mathrm{C} 3-\mathrm{C} 2-\mathrm{H} 2$ & 126.0 & $\mathrm{C} 17-\mathrm{C} 16-\mathrm{H} 16$ & 121.2 \\
\hline $\mathrm{C} 4-\mathrm{C} 3-\mathrm{C} 7$ & $117.2(2)$ & $\mathrm{C} 15-\mathrm{C} 16-\mathrm{H} 16$ & 121.2 \\
\hline $\mathrm{C} 4-\mathrm{C} 3-\mathrm{C} 2$ & $136.3(2)$ & $\mathrm{C} 16-\mathrm{C} 17-\mathrm{C} 18$ & $119.9(2)$ \\
\hline $\mathrm{C} 7-\mathrm{C} 3-\mathrm{C} 2$ & $106.46(19)$ & $\mathrm{C} 16-\mathrm{C} 17-\mathrm{H} 17$ & 120.0 \\
\hline $\mathrm{C} 5-\mathrm{C} 4-\mathrm{C} 3$ & $117.6(2)$ & $\mathrm{C} 18-\mathrm{C} 17-\mathrm{H} 17$ & 120.0 \\
\hline $\mathrm{C} 5-\mathrm{C} 4-\mathrm{H} 4$ & 121.2 & $\mathrm{~N} 5-\mathrm{C} 18-\mathrm{C} 17$ & $125.2(2)$ \\
\hline $\mathrm{C} 3-\mathrm{C} 4-\mathrm{H} 4$ & 121.2 & N5-C18-H18 & 117.4 \\
\hline $\mathrm{C} 4-\mathrm{C} 5-\mathrm{C} 6$ & $120.1(2)$ & $\mathrm{C} 17-\mathrm{C} 18-\mathrm{H} 18$ & 117.4 \\
\hline $\mathrm{C} 4-\mathrm{C} 5-\mathrm{H} 5$ & 119.9 & $\mathrm{~N} 5-\mathrm{C} 19-\mathrm{N} 4$ & $125.41(19)$ \\
\hline $\mathrm{C} 6-\mathrm{C} 5-\mathrm{H} 5$ & 119.9 & $\mathrm{~N} 5-\mathrm{C} 19-\mathrm{C} 15$ & $126.4(2)$ \\
\hline $\mathrm{N} 2-\mathrm{C} 6-\mathrm{C} 5$ & $125.0(2)$ & $\mathrm{N} 4-\mathrm{C} 19-\mathrm{C} 15$ & $108.23(18)$ \\
\hline $\mathrm{N} 2-\mathrm{C} 6-\mathrm{H} 6$ & 117.5 & $\mathrm{~N} 6-\mathrm{C} 20-\mathrm{C} 21$ & $124.8(3)$ \\
\hline $\mathrm{C} 5-\mathrm{C} 6-\mathrm{H} 6$ & 117.5 & $\mathrm{~N} 6-\mathrm{C} 20-\mathrm{H} 20$ & 117.6 \\
\hline $\mathrm{N} 2-\mathrm{C} 7-\mathrm{N} 1$ & $125.10(19)$ & $\mathrm{C} 21-\mathrm{C} 20-\mathrm{H} 20$ & 117.6 \\
\hline $\mathrm{N} 2-\mathrm{C} 7-\mathrm{C} 3$ & $126.6(2)$ & $\mathrm{C} 20-\mathrm{C} 21-\mathrm{C} 22$ & $119.7(2)$ \\
\hline $\mathrm{N} 1-\mathrm{C} 7-\mathrm{C} 3$ & $108.29(18)$ & $\mathrm{C} 20-\mathrm{C} 21-\mathrm{H} 21$ & 120.2 \\
\hline $\mathrm{N} 3-\mathrm{C} 8-\mathrm{C} 9$ & $124.4(3)$ & $\mathrm{C} 22-\mathrm{C} 21-\mathrm{H} 21$ & 120.2 \\
\hline $\mathrm{N} 3-\mathrm{C} 8-\mathrm{H} 8$ & 117.8 & $\mathrm{C} 21-\mathrm{C} 22-\mathrm{C} 23$ & $116.1(2)$ \\
\hline $\mathrm{C} 9-\mathrm{C} 8-\mathrm{H} 8$ & 117.8 & $\mathrm{C} 21-\mathrm{C} 22-\mathrm{C} 13$ & $122.5(2)$ \\
\hline $\mathrm{C} 8-\mathrm{C} 9-\mathrm{C} 10$ & $119.7(2)$ & $\mathrm{C} 23-\mathrm{C} 22-\mathrm{C} 13$ & $121.3(2)$ \\
\hline $\mathrm{C} 8-\mathrm{C} 9-\mathrm{H} 9$ & 120.1 & $\mathrm{C} 24-\mathrm{C} 23-\mathrm{C} 22$ & $119.7(2)$ \\
\hline $\mathrm{C} 10-\mathrm{C} 9-\mathrm{H} 9$ & 120.1 & $\mathrm{C} 24-\mathrm{C} 23-\mathrm{H} 23$ & 120.1 \\
\hline $\mathrm{C} 9-\mathrm{C} 10-\mathrm{C} 11$ & $116.3(2)$ & $\mathrm{C} 22-\mathrm{C} 23-\mathrm{H} 23$ & 120.1 \\
\hline $\mathrm{C} 9-\mathrm{C} 10-\mathrm{C} 1$ & $122.5(2)$ & $\mathrm{N} 6-\mathrm{C} 24-\mathrm{C} 23$ & $124.3(2)$ \\
\hline $\mathrm{C} 11-\mathrm{C} 10-\mathrm{C} 1$ & $121.1(2)$ & $\mathrm{N} 6-\mathrm{C} 24-\mathrm{H} 24$ & 117.8 \\
\hline $\mathrm{C} 12-\mathrm{C} 11-\mathrm{C} 10$ & $119.7(2)$ & $\mathrm{C} 23-\mathrm{C} 24-\mathrm{H} 24$ & 117.8 \\
\hline $\mathrm{C} 7-\mathrm{N} 1-\mathrm{C} 1-\mathrm{C} 2$ & $0.5(2)$ & $\mathrm{C} 19-\mathrm{N} 4-\mathrm{C} 13-\mathrm{C} 14$ & $-0.7(2)$ \\
\hline $\mathrm{C} 7-\mathrm{N} 1-\mathrm{C} 1-\mathrm{C} 10$ & $177.80(18)$ & $\mathrm{C} 19-\mathrm{N} 4-\mathrm{C} 13-\mathrm{C} 22$ & $-178.17(19)$ \\
\hline $\mathrm{N} 1-\mathrm{C} 1-\mathrm{C} 2-\mathrm{C} 3$ & $-0.2(2)$ & $\mathrm{N} 4-\mathrm{C} 13-\mathrm{C} 14-\mathrm{C} 15$ & $0.6(2)$ \\
\hline $\mathrm{C} 10-\mathrm{C} 1-\mathrm{C} 2-\mathrm{C} 3$ & $-177.3(2)$ & $\mathrm{C} 22-\mathrm{C} 13-\mathrm{C} 14-\mathrm{C} 15$ & $177.8(2)$ \\
\hline $\mathrm{C} 1-\mathrm{C} 2-\mathrm{C} 3-\mathrm{C} 4$ & $179.9(2)$ & $\mathrm{C} 13-\mathrm{C} 14-\mathrm{C} 15-\mathrm{C} 16$ & $-179.2(3)$ \\
\hline $\mathrm{C} 1-\mathrm{C} 2-\mathrm{C} 3-\mathrm{C} 7$ & $-0.1(2)$ & $\mathrm{C} 13-\mathrm{C} 14-\mathrm{C} 15-\mathrm{C} 19$ & $-0.3(2)$ \\
\hline $\mathrm{C} 7-\mathrm{C} 3-\mathrm{C} 4-\mathrm{C} 5$ & $0.3(3)$ & $\mathrm{C} 19-\mathrm{C} 15-\mathrm{C} 16-\mathrm{C} 17$ & $0.5(3)$ \\
\hline $\mathrm{C} 2-\mathrm{C} 3-\mathrm{C} 4-\mathrm{C} 5$ & $-179.7(2)$ & $\mathrm{C} 14-\mathrm{C} 15-\mathrm{C} 16-\mathrm{C} 17$ & $179.3(2)$ \\
\hline $\mathrm{C} 3-\mathrm{C} 4-\mathrm{C} 5-\mathrm{C} 6$ & $0.0(4)$ & $\mathrm{C} 15-\mathrm{C} 16-\mathrm{C} 17-\mathrm{C} 18$ & $-0.4(4)$ \\
\hline $\mathrm{C} 7-\mathrm{N} 2-\mathrm{C} 6-\mathrm{C} 5$ & $0.4(3)$ & $\mathrm{C} 19-\mathrm{N} 5-\mathrm{C} 18-\mathrm{C} 17$ & $0.0(3)$ \\
\hline $\mathrm{C} 4-\mathrm{C} 5-\mathrm{C} 6-\mathrm{N} 2$ & $-0.3(4)$ & $\mathrm{C} 16-\mathrm{C} 17-\mathrm{C} 18-\mathrm{N} 5$ & $0.1(4)$ \\
\hline $\mathrm{C} 6-\mathrm{N} 2-\mathrm{C} 7-\mathrm{N} 1$ & $179.2(2)$ & $\mathrm{C} 18-\mathrm{N} 5-\mathrm{C} 19-\mathrm{N} 4$ & $-179.1(2)$ \\
\hline $\mathrm{C} 6-\mathrm{N} 2-\mathrm{C} 7-\mathrm{C} 3$ & $-0.1(3)$ & $\mathrm{C} 18-\mathrm{N} 5-\mathrm{C} 19-\mathrm{C} 15$ & $0.2(3)$ \\
\hline $\mathrm{C} 1-\mathrm{N} 1-\mathrm{C} 7-\mathrm{N} 2$ & $-179.9(2)$ & $\mathrm{C} 13-\mathrm{N} 4-\mathrm{C} 19-\mathrm{N} 5$ & $180.0(2)$ \\
\hline $\mathrm{C} 1-\mathrm{N} 1-\mathrm{C} 7-\mathrm{C} 3$ & $-0.5(2)$ & $\mathrm{C} 13-\mathrm{N} 4-\mathrm{C} 19-\mathrm{C} 15$ & $0.6(2)$ \\
\hline
\end{tabular}




\begin{tabular}{ll}
$\mathrm{C} 4-\mathrm{C} 3-\mathrm{C} 7-\mathrm{N} 2$ & $-0.2(3)$ \\
$\mathrm{C} 2-\mathrm{C} 3-\mathrm{C} 7-\mathrm{N} 2$ & $179.8(2)$ \\
$\mathrm{C} 4-\mathrm{C} 3-\mathrm{C} 7-\mathrm{N} 1$ & $-179.60(18)$ \\
$\mathrm{C} 2-\mathrm{C} 3-\mathrm{C} 7-\mathrm{N} 1$ & $0.4(2)$ \\
$\mathrm{C} 12-\mathrm{N} 3-\mathrm{C} 8-\mathrm{C} 9$ & $-0.9(4)$ \\
$\mathrm{N} 3-\mathrm{C} 8-\mathrm{C} 9-\mathrm{C} 10$ & $0.8(4)$ \\
$\mathrm{C} 8-\mathrm{C} 9-\mathrm{C} 10-\mathrm{C} 11$ & $0.2(4)$ \\
$\mathrm{C} 8-\mathrm{C} 9-\mathrm{C} 10-\mathrm{C} 1$ & $-178.6(2)$ \\
$\mathrm{C} 2-\mathrm{C} 1-\mathrm{C} 10-\mathrm{C} 9$ & $170.0(2)$ \\
$\mathrm{N} 1-\mathrm{C} 1-\mathrm{C} 10-\mathrm{C} 9$ & $-6.7(3)$ \\
$\mathrm{C} 2-\mathrm{C} 1-\mathrm{C} 10-\mathrm{C} 11$ & $-8.7(4)$ \\
$\mathrm{N} 1-\mathrm{C} 1-\mathrm{C} 10-\mathrm{C} 11$ & $174.5(2)$ \\
$\mathrm{C} 9-\mathrm{C} 10-\mathrm{C} 11-\mathrm{C} 12$ & $-0.9(3)$ \\
$\mathrm{C} 1-\mathrm{C} 10-\mathrm{C} 11-\mathrm{C} 12$ & $177.9(2)$ \\
$\mathrm{C} 8-\mathrm{N} 3-\mathrm{C} 12-\mathrm{C} 11$ & $0.0(4)$ \\
$\mathrm{C} 10-\mathrm{C} 11-\mathrm{C} 12-\mathrm{N} 3$ & $0.9(4)$ \\
\hline
\end{tabular}

$-0.5(3)$

$-179.6(2)$

$178.96(19)$

$-0.2(2)$

$0.4(4)$

$-0.3(4)$

$-0.1(3)$

$178.4(2)$

$-168.3(2)$

$8.6(3)$

$10.1(3)$

$-173.0(2)$

$0.2(3)$

$-178.2(2)$

-0.2 (4)

$-0.1(4)$

Hydrogen-bond geometry $\left(A,{ }^{\circ}\right)$

\begin{tabular}{lllll}
\hline$D-\mathrm{H} \cdots A$ & $D-\mathrm{H}$ & $\mathrm{H} \cdots A$ & $D \cdots A$ & $D-\mathrm{H} \cdots A$ \\
\hline $\mathrm{N} 1-\mathrm{H} 1 \cdots \mathrm{N} 2^{\mathrm{i}}$ & 0.86 & 2.22 & $3.061(3)$ & 167 \\
$\mathrm{~N} 4-\mathrm{H} 4 A \cdots \mathrm{N} 5^{\mathrm{ii}}$ & 0.86 & 2.22 & $3.066(3)$ & 169 \\
\hline
\end{tabular}

Symmetry codes: (i) $-x,-y+2,-z+2$; (ii) $-x+1,-y+2,-z+1$. 